\title{
La zone pellucide bovine : différences de composition macromoléculaire entre ovocytes, prétraités ou non à l'A 23187, et embryons
}

\author{
S Bercegeay 1, F Allaire 2, M Jean 1, A L'Hermite 1, JF Bruyas 2, \\ N Renard 2, D Tainturier 2, P Barrière 1 \\ ${ }^{1}$ CHU-HME, laboratoire de Biologie de la reproduction, 44035 Nantes Cedex; \\ 2 École nationale vétérinaire de Nantes, laboratoire de Pathologie de la reproduction, \\ 44026 Nantes Cedex, France
}

(Reçu le 23 février 1993; accepté le 17 septembre 1993)

Résumé - Nous avons confirmé la présence, au niveau de la zone pellucide bovine (ZP), de 3 types de glycoprotéines nommées ZP1, ZP2, ZP3 de masse moléculaire apparente respective de 80-70 kDa, 66-63 kDa et $60 \mathrm{kDa}$, après séparation en SDS-PAGE et en conditions réductrices. En 2D-PAGE, le profil électrophorétique fait apparaître une quatrième famille de glycoprotéines appelée ZP4. Les analyses des constituants protéiques de ZP d'ovocytes et d'embryons obtenus après transit dans le tractus génital, et/ou après action du ionophore A23187, montrent des modifications de leur composition macromoléculaire illustrées par des mobilités électrophorétiques caractéristiques.

zone pellucide / bovin / SDS-PAGE / électrofocalisation (IEF) / 2D-PAGE

Summary - Differences in the macromolecular composition of zona pellucida isolated from bovine oocytes, embryos and A23187 pretreated oocytes. The SDS-PAGE method was used to determine the composition of isolated bovine zona pellucida (ZP). This egg extracellular coat appears to be characterized by 3 major glycoproteins (ZP1, ZP2, ZP3), with apparent molecular weight (MW) of 80-70 kD, 66-63 kD and $60 \mathrm{kD}$, respectively, as revealed by 1-dimensional SDS-PAGE. After 2-dimensional SDS-PAGE, the zona pellucida electrophoretic pattern indicates a fourth glycoprotein, thus called ZP4. SDS-PAGE analysis of ZP isolated from oocytes and embryos after transit through the genital tract of A23187 pretreated oocytes allowed the description of modifications in glycoproteinic composition.

zona pellucida / bovine / SDS-PAGE / IEF / 2D-PAGE 


\section{INTRODUCTION}

La zone pellucide (ZP) enveloppe acellulaire entourant l'ovocyte des Mammifères (Dunbar, 1983) est impliquée dans la reconnaissance homospécifique, la fixation et le déclenchement de la réaction acrosomique du spermatozoïde (Hartmann, 1983 ; Barros et al, 1988 ; Wassarman et Mortillo, 1991).

Après la fécondation, la ZP participe au blocage de la polyspermie (StewartSavage and Bavister, 1988) et assure la protection mécanique de l'embryon préimplantatoire (Mintz, 1962; Modlinski, 1970).

Du fait de ses rôles importants, la ZP présente un grand intérêt dans l'étude de la reproduction, notamment comme cible pour l'immuno-contraception (Gwatkin et al, 1977 ; Isojima et al, 1984 ; Mori et al, 1985 ; Koyama et al, 1991). Cette structure principalement constituée de glycoprotéines a été bien caractérisée dans plusieurs espèces animales (Dunbar, 1983; Dunbar et Wolgemuth, 1984 ; Hedrick et Wardrip, 1987a ; Wassarman, 1988).

Ces glycoprotéines sont sécrétées par l'ovocyte seul ou en coopération avec les cellules folliculaires (Bleil et Wassarman, 1980a; Greve et al, 1982 ; Wolgemuth et al, 1984 ; Dunbar et al, 1989). L'oviducte est aussi impliqué dans la synthèse des composants de la ZP (Kapur and Johnson, 1988 ; Gandolfi et al, 1991 ; Boice et al, 1990) et, lors de la fécondation, la ZP subit des modifications physiques et chimiques.

Cette structure est soumise à de multiples changements biochimiques et structuraux au cours de son existence.

Le but de ce travail est de caractériser, par électrophorèse SDS-PAGE et en 2 dimensions, les glycoprotéines constitutives de la ZP bovine et de mettre en évidence d'éventuelles modifications liées au transit dans l'oviducte, à la réaction corticale et à la fécondation.

\section{MATÉRIEL ET MÉTHODES}

\section{Recueil des ZP}

Le recueil a été effectué à partir de 3 groupes différents.

Des ovaires $(n=160)$ sont prélevés à l'abattoir et conservés sur de la glace pendant 2 à $3 \mathrm{~h}$. Les ovocytes sont obtenus par ponction individuelle à $+4^{\circ} \mathrm{C}$ des follicules pré-antraux (diamètre supérieur à $8 \mathrm{~mm}$ ), à l'aide d'une aiguille de $0,8 \mathrm{~mm}$ de diamètre reliée à un système d'aspiration. Le liquide de ponction est ensuite passé sur 2 filtres successifs de nylon (maille de $150 \mu \mathrm{m}$ puis $75 \mu \mathrm{m}$ ). Les ovocytes sont remis en suspension dans une boîte de Petri après lavage des filtres avec du $\mathrm{NaCl}$ à $0,9 \%$ à $+4^{\circ} \mathrm{C}$. Les ovocytes recueillis sont enfin rincés par 3 passages successifs dans des bains de $\mathrm{NaCl}$ à $0,9 \%$.

Les embryons et ovocytes ovulés sont obtenus après superovulation de 14 vaches après des chaleurs induites par la pose d'implants sous-cutanés contenant un analogue de la progestérone: le norgestomet (Crestar, Intervet). Les ovaires sont stimulés par injections intramusculaires de $\mathrm{FSH}$ (Stimufol) au $7^{\mathrm{e}}$ jour après la pose de l'implant à la dose de $32 \mathrm{mg}$ réparties en quantités décroissantes sur $4 \mathrm{j}$ à $12 \mathrm{~h}$ d'intervalle afin d'imiter la cinétique de FSH pendant le cycle ostral naturel. Chacune des cornes utérines est lavée avec 300 à $500 \mathrm{ml}$ de liquide de collecte $(2 \%$ de sérum de bœuf dans du sérum physiologique) $7 \mathrm{j}$ après l'ovulation à l'aide d'une sonde de récolte par voie cervicale. Dix vaches inséminées avec du sperme décongelé ont fourni 40 embryons au stade soit morula soit blastocyste ; 4 vaches non inséminées ont permis l'obtention de 43 ovocytes non fécondés. Les embryons et ovocytes sont également lavés 3 fois dans du $\mathrm{NaCl}$ à $0,9 \%$.

\section{Isolement et solubilisation des ZP}

Les ovocytes ponctionnés sont débarrassés des ceilules périovocytaires par agitation dans un 
tampon de lavage (phosphate de sodium 10 $\mathrm{mM}$, chlorure de sodium $125 \mathrm{mM}$, citrate de sodium $3 \mathrm{mM}$, EDTA $2 \mathrm{mM}$ ) (Hedrick and Wardrip, 1986). Quelque 1500 ovocytes, colorés au bleu d'aniline (bleu lumière, Prolabo, France) pour mieux les visualiser, sont transférés dans de l'eau distillée de façon à exercer par osmose une pression sur la ZP. L'agitation mécanique du milieu à l'aide d'une micropipette provoque la rupture de la ZP et l'extrusion du contenu ovocytaire. Pour éviter la protéolyse des constituants de la ZP, l'ensemble des manipulations est réalisé à $+4^{\circ} \mathrm{C}$ en présence d'inhibiteurs enzymatiques (protease inhibitors set: Boehringer Mannheim Biochemica, France: dihydrochlorure d'antipaîne $50 \mu \mathrm{g} / \mathrm{ml}$, Aprotinine $2 \mu \mathrm{g} / \mathrm{ml}$, EDTA$\mathrm{Na}_{2} 0,5 \mathrm{mg} / \mathrm{ml}$, leupeptine $0,5 \mu \mathrm{g} / \mathrm{ml}$, phosphoramidon $330 \mu \mathrm{g} / \mathrm{ml}$ ). Les ZP sont lavées par passages successifs dans 3 bains de $\mathrm{NaCl}$ à $0,9 \%$ puis solubilisées à $70^{\circ} \mathrm{C}$ pendant 30 min dans de l'eau dont le $\mathrm{pH}$ a été ajusté à 10,0 avec du $\mathrm{Na}_{2} \mathrm{CO}_{3}$ solide (Dunbar et al, 1980). L'échantillon est concentré à $45^{\circ} \mathrm{C}$ sous vide à l'aide d'un concentrateur Speed-Vac (Savant, Bioblock, France) puis stocké à $-20^{\circ} \mathrm{C}$ et utilisé dans le mois.

Un second protocole plus rapide a été établi pour le traitement de grandes quantités d'ovocytes. La solubilisation des ZP s'effectue à partir d'ovocytes entiers (Araki et al, 1992). Cinq cents ovocytes sont disposés dans un tube Eppendorf contenant $500 \mu \mathrm{l}$ d'eau distillée à pH 10,0. Chaque tube est ensuite incubé à $70^{\circ} \mathrm{C}$ pendant 30 à $60 \mathrm{~min}$. L'état de solubilisation de la ZP est contrôlé à un grossissement 40 à l'aide d'un stéréomicroscope. Après centrifugation à $14300 \mathrm{~g}$ pendant 20 minutes afin de sédimenter ovocytes et particules insolubles, le surnageant est concentré et stocké à $-20^{\circ} \mathrm{C}$.

\section{Action d'un ionophore calcique : A 23187}

Un lot d'ovocytes $(n=1000)$ est transféré, après ponction, dans $450 \mu \mathrm{l}$ de milieu de culture (INRA Ménézo $B_{2}$ Medium, Biomérieux, France) puis soumis à l'action de $50 \mu \mathrm{l}$ de l'ionophore $\mathrm{A}$ $23187\left(25 \mu \mathrm{M}\right.$ dans PBS) durant $15 \mathrm{~min}$ à $37^{\circ} \mathrm{C}$ sous $5 \%$ de $\mathrm{CO}_{2}$. Selon Moller et Wassarman (1989), l'action de l'ionophore doit déclencher la réaction corticale et par conséquent la réaction de la zone pellucide. Les ovocytes sont ensuite rincés par bains successifs dans du $\mathrm{NaCl}$ à $0,9 \%$ puis séparés de leur ZP selon la technique décrite ci-dessus. Parallèlement, un échantillon témoin est traité de la même façon sans ionophore. Les ZP solubilisées sont con-servées à $-20^{\circ} \mathrm{C}$.

\section{Méthodes électrophorétiques}

SDS-PAGE : les ZP solubilisées sont mélangées avec 3 volumes d'une solution dénaturante $(0,0625 \mathrm{M}$ tris $\mathrm{pH} 6,8$, contenant $20 \%$ de glycérol, $5 \%$ de $\beta$-mercaptoéthanol, $2 \%$ de SDS et $0,001 \%$ de bleu de Bromophénol). L'échantillon est chauffé dans un bain-marie à $95^{\circ} \mathrm{C}$ pendant $5 \mathrm{~min}$. La séparation électrophorétique s'effectue sur gel de polyacrylamide en présence de SDS (Laemmli, 1970) à voltage constant $1 \mathrm{~h}$ à température ambiante.

Le gel de concentration contient $4 \%(\mathrm{p} / \mathrm{v})$ d'acrylamide, le gel de séparation est constitué d'un gradient linéaire de 6 à $16 \%(\mathrm{p} / \mathrm{v})$ d'acrylamide.

2D-PAGE ; la technique adoptée est celle décrite par O'Farrell (1975). Les ZP solubilisées et mélangées à 3 volumes de tampon de lyse (urée 9,5 M, ampholines pH 3,5-10 2\% Resolyte, Touzart et Matignon, France, NP 40 2\%, $\beta$ mercaptoéthanol $5 \%$ ) sont séparées dans un premier temps selon leur point isoélectrique par électrofocalisation (IEF) puis en fonction de leur poids moléculaire par SDS-PAGE dans un gel de 6 à $16 \%(\mathrm{p} / \mathrm{v})$ d'acrylamide.

Les concentrations protéiques sont déterminées en utilisant l'acide bicinchoninique ( $\mathrm{BCA}$ ) (Smith et al, 1985). Environ $200 \mathrm{ng}$ de protéines sont déposés par puits de migration en SDSPAGE et $4 \mu \mathrm{g}$ pour l'IEF.

Le liquide folliculaire, source possible de contamination protéique des ZP, est également soumis aux migrations à raison de $6 \mu \mathrm{g} /$ puits.

Les masses moléculaires (MM) sont estimées par comparaison avec les mobilités relatives de plusieurs protéines de poids moléculaire connu: SDS Molecular Weight Standards, Biorad: phosphorylase $b$ du muscle de lapin $(\mathrm{MM}=97,4 \mathrm{kDa}), \mathrm{BSA}(\mathrm{MM}=66,2 \mathrm{kDa})$, ovalbumine d'œuf de poule ( $\mathrm{MM}=45,0 \mathrm{kDa})$, anhydrase carbonique bovine (MM $=31,0 \mathrm{kDa})$, inhibiteur trypsique de graine de haricot $(\mathrm{MM}=21,5$ $\mathrm{kDa})$, lysozyme d'œuf de poule $(\mathrm{MM}=14,4$ $\mathrm{kDa})$. 
De la même façon, les points isoélectriques (PI) sont déterminés à l'aide de marqueurs : 2DSDS-PAGE Standards, Biorad: conalbumine d'œuf de poule ( $P I=6,0-6,3-6,6)$, actine de muscle bovin ( $P I=5,0-5,1$ ), anhydrase carbonique bovine ( $P \mid=5,9-6,0)$, inhibiteur trypsique de graine de haricot $(P I=4,5)$. Les protéines sont colorées à l'argent selon la méthode de Morrissey (1981). Malgré sa faible sensibilité, une coloration préalable des gels à l'acide périodique de Schiff (Zacharius et al, 1969) a confirmé la nature glycoprotéique des molécules constitutives de la ZP (non illustré).

\section{RÉSULTATS}

La séparation en SDS-PAGE de première dimension et en conditions réductrices des protéines de ZP d'ovocytes ponctionnés montre, après coloration à l'argent, 3 bandes localisées à des MM apparentes comprises entre 80-70 kDa, 66-63 kDa et à $60 \mathrm{kDa}$ nommées respectivement $\mathrm{ZP} 1$, ZP2 et ZP3 selon le système d'identification suivant les $M M$ apparentes décroissantes proposé par Sacco et al (1981) (fig 1).

Lorsque les ZP sont issues d'ovocytes ou d'embryons ayant transité dans le tractus génital, les mêmes conditions de séparation font apparaître 2 bandes supplémentaires avec des MM respectives vers $97 \mathrm{kDa}$ et $45 \mathrm{kDa}$ (fig 2). Le traitement à I'A 23187 ne modifie pas le profil de migration.

La séparation en 2 dimensions met en évidence 4 familles de glycoprotéines acides qui, selon le système d'identification des MM apparentes décroissantes, sont appelées : ZP1 (MM $=117-97 \mathrm{kDa}$; $\mathrm{PI}=5,4-6,3), \mathrm{ZP} 2(\mathrm{MM}=100-60 \mathrm{kDa} ; \mathrm{PI}$ $=4,3-5,6)$, ZP3 $(\mathrm{MM}=90-74 \mathrm{kDa} ; \mathrm{PI}=$ $5,8-6,2)$ et $\mathrm{ZP} 4(\mathrm{MM}=60-55 \mathrm{kDa} ; \mathrm{PI}=$ 5,6-6,2) (fig 3A). Après prétraitement des ovocytes par un ionophore A 23187 (25 $\mu \mathrm{M}$ dans $\mathrm{PBS})$, le profil électrophorétique est modifié. ZP1 présente dans ce cas une

\section{WM \\ (kD)}

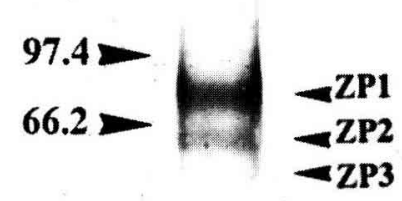

45.0

\section{$31.0=$}

\section{$21.5=$ \\ 14.4}

Fig 1. Séparation des protéines de la ZP d'ovocytes ovariens bovins en électrophorèse monodimensionnelle et en conditions réductrices (environ $200 \mathrm{ng}$ de protéines sont déposés par puits). Coloration à l'argent.

MM apparente comprise entre 182 et 135 $\mathrm{kDa}(\mathrm{PI}=4,9-5,4)$ et $\mathrm{ZP2}$ une MM apparente entre 115 et $55 \mathrm{kDa}(\mathrm{PI}=4,1-5,6)$, ZP3 et ZP4 n'apparaissent plus au sein du gel (fig $3 C$ ).

\section{DISCUSSION}

Depuis ces 10 dernières années, de nombreuses études concernant la ZP de plusieurs espèces de Mammifères ont été réalisées. Si le bovin constitue un matériel de choix pour l'étude de la ZP en raison de la facilité d'obtention du matériel, le nombre de travaux reste limité.

La ZP apparaît après séparation en SDS-PAGE, composée de 3 familles de glycoprotéines nommées ZP1 (80-70 kDa), 


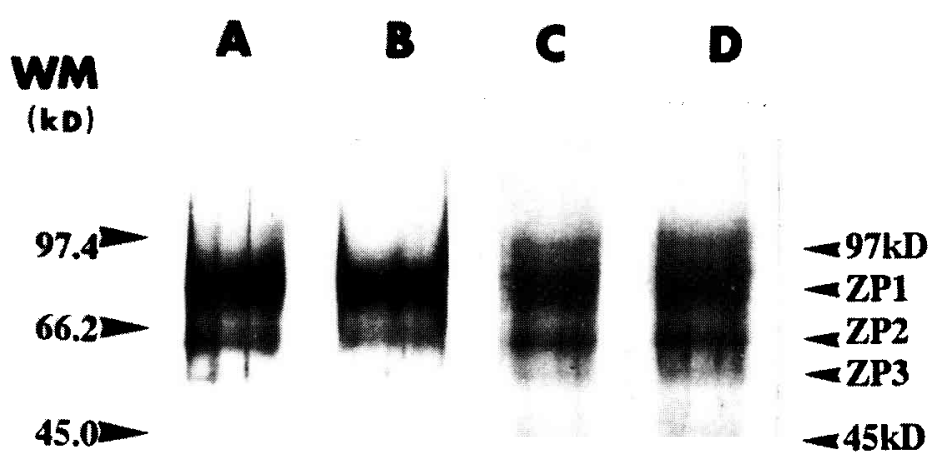

\section{$31.0=$}

\section{5 - \\ $14.4=$}

Fig 2. Comparaison des profils de migration électrophorétique en première dimension et en conditions réductrices. A : ZP d'ovocytes ponctionnés; B : ZP d'ovocytes ponctionnés puis soumis au ionophore A 23187 (25 $\mu \mathrm{M}$ dans PBS) ; C : ZP d'embryons à $\mathrm{J} 7$; D : ZP d'ovocytes à J7 prélevés chez des vaches non inséminées. Coloration à l'argent.

ZP2 (66-63 kDa), ZP3 (60 kDa) selon le système d'identification proposé par Sacco et al (1981). Si les MM apparentes sont comparables à celles observées dans diverses espèces (Bleil and Wassarman, 1980b; Sacco et al, 1981 ; Dunbar et al, 1985 ; Maresh and Dunbar, 1987 ; Shabanowitz and O-Rand, 1988), ces résultats ne sont pas en accord avec ceux obtenus par Gwatkin et al (1980) qui, après élimination des cellules périovocytaires par la collagénase $\left(100 \mu \mathrm{g} / \mathrm{ml}\right.$ à $37^{\circ} \mathrm{C}$ pendant $\left.2 \mathrm{~h}\right)$, séparent les glycoprotéines sur gel d'agarose $(1 \%)$ en une unique bande localisée vers $100 \mathrm{kDa}$ chez le bovin et $62 \mathrm{kDa}$ chez porc. Dunbar et al (1980), en SDS-PAGE, n'avaient observé qu'une unique bande de 40 à $60 \mathrm{kDa}$ chez la vache comme chez le porc, alors qu'il est désormais établi que les glycoprotéines de ZP de porc se sépa- rent, en SDS-PAGE en conditions réductrices, en 4 familles de $M M$ apparentes de $90 \mathrm{kDa}, 65 \mathrm{kDa}, 55 \mathrm{kDa}$ et $25 \mathrm{kDa}$ (Wassarman, 1988).

La deuxième dimension fait apparaître 4 familles de protéines acides : ZP1 ( $M M=$ 117-97 $\mathrm{kDa} ; \mathrm{PI}=5,4-6,3), \mathrm{ZP2}(\mathrm{MM}=$ $100-60 \mathrm{kDa} ; \mathrm{PI}=4,3-5,6), \mathrm{ZP} 3(\mathrm{MM}=90-$ $74 \mathrm{kDa} ; \mathrm{PI}=5,8-6,2)$ et $\mathrm{ZP4}(\mathrm{MM}=60$ $55 \mathrm{kDa}$; $\mathrm{PI}=5,6-6,2)$ selon le système d'identification suivant les MM apparentes décroissantes (Sacco et al, 1981). Le profil électrophorétique obtenu est proche de celui observé par Florman et First (1988), qui cependant ne montre que 3 trains de protéines présentant chacun une microhétérogénéité prononcée et de $\mathrm{MM}$ apparente de 94-110 $\mathrm{kDa}(\mathrm{PI}=7,0-8,3), 82-94$ $\mathrm{kDa}(\mathrm{PI}=4,3-4,8)$ et $70-76 \mathrm{kDa}(\mathrm{PI}=5,8$ $7,3)$. Au cours du recueil des $Z P$, I'utilisa- 
tion de hyaluronidase ( $1 \mathrm{mg} / \mathrm{ml}$ ) (Type IV ; Sigma) et l'absence d'inhibiteurs enzymatiques lors de l'éclatement des ZP et de l'extrusion du contenu ovocytaire, pourrait modifier les PI des constituants de la ZP et une protéolyse de la $4^{e}$ famille pourrait expliquer son absence au sein du gel.

La caractérisation en SDS-PAGE des ZP d'embryons ou ovocytes non inséminés, prélevés par lavages utérins $7 \mathrm{j}$ après ovulation, fait apparaître 2 nouvelles bandes dont les MM apparentes se situent respectivement vers $97 \mathrm{kDa}$ et $45 \mathrm{kDa}$.

L'existence de glycoprotéines secrétées par des cellules de l'oviducte et associées à la ZP lors du transit de l'œuf dans le tractus a été décrite chez le porc (Hedrick et al, 1987b), le lapin (Shapiro et al, 1974), la souris (Kapur and Johnson, 1988), le babouin (Boice et al, 1990), la brebis (Gandolfi et al, 1991) et chez le hamster (Roux et Kan, 1991; Abe et al, 1992). Chez le bovin, le temps moyen de dissolution de la ZP par la pronase à $0,1 \%$ augmente avec le temps de séjour de l'ovocyte dans un oviducte isolé (Smorag and Katska, 1988). De plus, une protéine de $97 \mathrm{kDa}$ sécrétée au niveau de l'isthme et capable de s'associer avec la ZP a été mise en évidence en immunofluorescence par Wegner et Killian (1991). Cette protéine, nécessaire à la régulation des étapes initiales de la fécondation et/ou du développement précoce, est présente sur le profil électrophorétique de l'embryon et de l'ovocyte ovulé (fig 2). La seconde protéine localisée à $45 \mathrm{kDa}$ est plus faiblement représentée. Elle pourrait être issue
- de la même façon - de sécrétions de cellules de l'oviducte ou d'un clivage des glycoprotéines sous l'action d'enzymes présentes dans le trompes. II n'est cependant pas observé, corrélativement, d'atténuation ou de disparition des bandes propres aux glycoprotéines d'ovocytes ovariens.

En SDS-PAGE, aucune différence n'est décelable entre ZP d'ovocytes ovulés et ZP d'embryons à J7 et, ZP d'ovocytes ponctionnés et ZP d'ovocytes prétraités par le ionophore (fig 2). II est pourtant connu chez le bovin que la fécondation provoque un durcissement (Hardening) de la ZP alors moins sensible à l'action de protéases et d'agents chimiques (Katska et al, 1989).

Chez la souris, la réaction corticale conduit au clivage de la glycoprotéine ZP2 en ZP2 $f$ de MM apparente plus faible. Cette protéolyse serait impliquée dans le blocage de la polyspermie (Moller and Wassarman, 1989). De la même façon, ZP3 est modifiée en ZP3 $f$ incapable de se fixer aux spermatozoïdes; cependant cette modification est indétectable en SDS-PAGE. Une évaluation plus résolutive des constituants de la $\mathrm{ZP}$ en $2 \mathrm{D}$ a permis la mise en évidence des modifications dans la composition macromoléculaire de ZP d'ovocytes prétraités à l'A 23187. ZP1 est alors représentée par un spot localisé à une $\mathrm{MM}$ apparente comprise entre 182 et $135 \mathrm{kDa}$ avec un $\mathrm{Pl}$ de 4,9-5,4. ZP 2 semble être le résultat de fusion avec d'autres trains protéiques. Cette famille apparaît plus étalée $(\mathrm{PI}=4,1-5,6)$ avec une

Fig 3. Séparation des protéines de la ZP en 2 dimensions et en conditions réductrices après solubilisation à partir d'ovocytes entiers. Les ZP solubilisées sont mélangées à 3 volumes d'un tampon de lyse (urée 9,5 M, ampholines [3,5-10] 2\%, NP $402 \%, \beta$-mercaptoéthanol $5 \%$ ). A : ZP d'ovocytes ponctionnés ; $\mathrm{B}: \mathrm{ZP}$ d'ovocytes ponctionnés puis incubés $15 \mathrm{~min}$ à $37^{\circ} \mathrm{C}$ sous $5 \%$ de $\mathrm{CO}_{2}$ dans du $\mathrm{B}_{2}$. $\mathrm{C}: \mathrm{ZP}$ d'ovocytes ponctionnés puis incubés $15 \mathrm{~min}$ à $37^{\circ} \mathrm{C}$ sous $5 \%$ de $\mathrm{CO}_{2}$ dans du $\mathrm{B}_{2}$ en présence du ionophore A 23187 ( $25 \mu \mathrm{M}$ dans du PBS) (4 $\mu \mathrm{g}$ de protéines). Coloration à l'argent. 


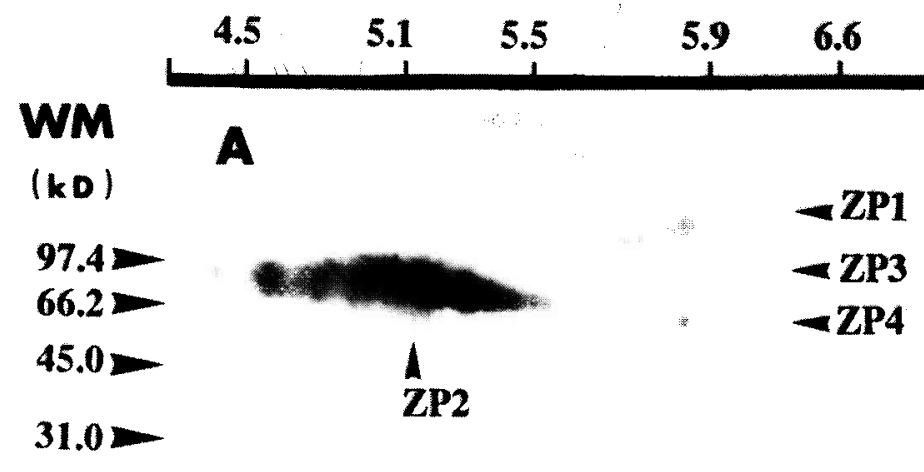

21.5 =

14.4 =
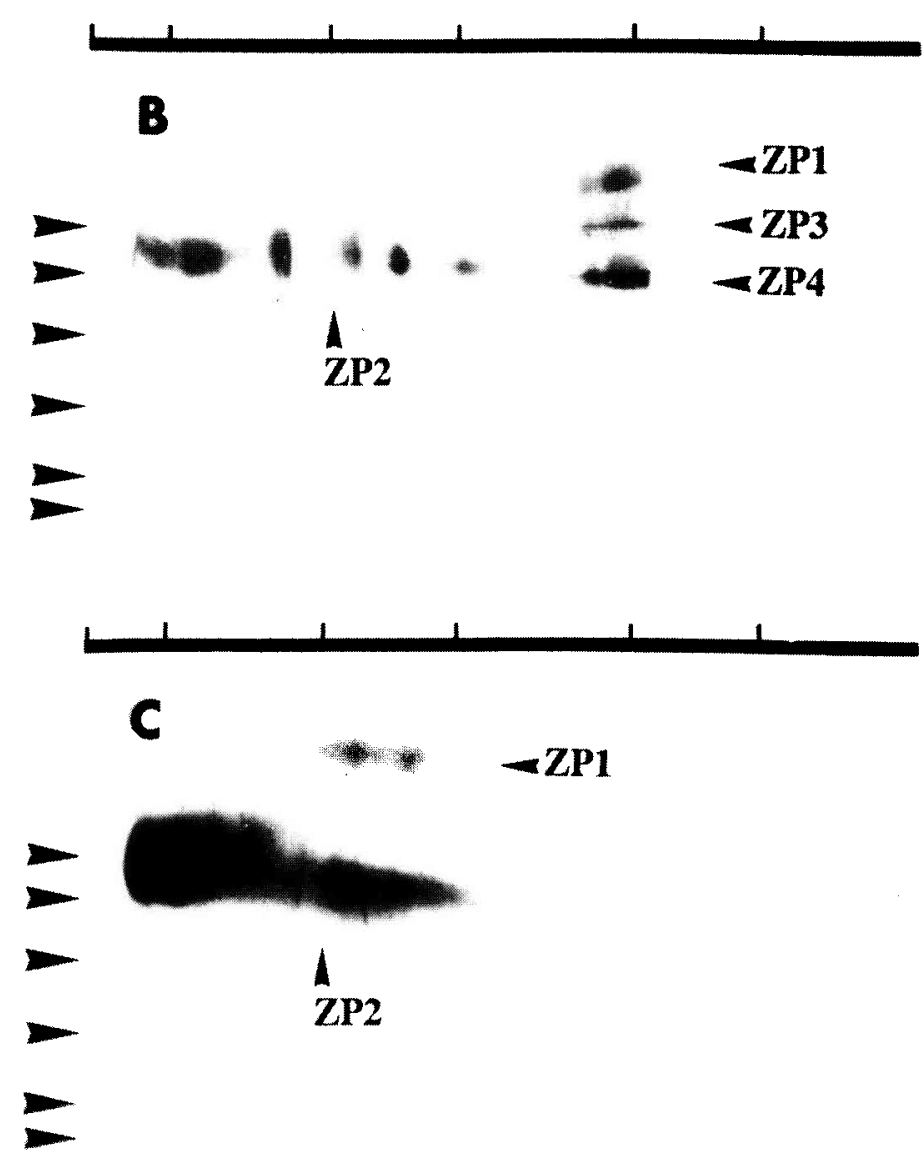
MM apparente entre 115 et 55 kDa corrélativement à la disparition de ZP3 et ZP4. Le contenu des granules corticaux pourrait agir plus spécifiquement sur les séquences saccharidiques et expliquer ainsi les variations de PI observées. Chez le xénope, une $\beta-\mathrm{N}$-acétylglucosaminidase a été identifiée dans les granules corticaux (Greve et al, 1985). L'action de protéases conduirait à un clivage protéique ayant pour résultat la disparition d'une part des protéines. De plus, l'action des enzymes peut modifier l'encombrement stérique des molécules, directement lié à la valeur des MM apparentes.

\section{CONCLUSION}

La zone pellucide bovine est composée de 3 à 5 glycoprotéines dont certaines ont des mobilités électrophorétiques caractéristiques pouvant avoir valeur de marqueurs de compétences fonctionnelles de la ZP selon qu'elle provient :

- i) d'ovocytes préovulatoires, d'ovocytes qui ont subi la réaction corticale ou d'embryons préimplantatoires. Ces modifications peuvent traduire l'action sur la ZP des sécrétions tubaires et des granules corticaux dont les facteurs et le mode d'action restent à élucider ;

- ii) de l'espèce, puisque la ZP bovine diffère de la ZP de souris en dépit de certaines similitudes, du fait possible de la spécificité des intéractions gamétiques.

\section{RÉFÉRENCES}

Abe H, Ookata K, Abe M, Oïkawa T (1992) Immunological characterization of oviductal glycoproteins associated with the zona pellucida of the golden hamster. J Exp Zool 262, 203-218

Araki Y, Orgebin-Crist MC, Tulsiani DRP (1992) Qualitative characterization of oligosaccha- ride chain present on the rat zona pellucida glycoconjugates. Biol Reprod 4, 912-919

Barros C, Jedlicki A, Vigil P (1988) The gamete membrane fusion test to assay the fertilizing ability of human spermatozoa. Human $R e-$ prod 3, 637-644

Bleil JD, Wassarman PM (1980a) Synthesis of zone pellucida proteins by denuded and follicle-enclosed mouse oocytes during culture in vitro. Proc Natl Acad Sci (USA) 70, 10291033

Bleil JD, Wassarman PM (1980b) Mammalian sperm-egg interaction: identification of a glycoprotein in mouse egg zonae pellucidae possessing receptor activity for sperm. Cell 20, 873-882

Boice MJ, Mc Carthy TJ, Mavrogianis PA, Fazleabas A, Verhage HG (1990) Localization of oviductal glycoproteins within the zona pellucida and perivitelline space of ovulated ova and early embryos in baboons (Papio anubis). Biol Reprod 43, 340-346

Dunbar BS (1983) Morphological, biochemical and immunochemical characterization of the mammalian zona pellucida. In: Mechanism and Control of Animal Fertilization (JF Hartman, ed) Academic Press, New York, 139175

Dunbar BS, Wolgemuth DJ (1984) Structure and function of the mammalian zona pellucida, a unique extra-cellular matrix. In: Modern Cell Biology (R Alan, ed). Liss Inc, New York, 77111

Dunbar BS, Wardrip NJ, Hedrick JL (1980) Isolation, physicochemical properties, and macromolecular composition of zona pellucida from porcine oocytes. Biochemistry 19, 356365

Dunbar BS, Dudkiewicz A, Bundman DS (1985) Proteolysis of specific porcine zone pellucida glycoproteins by boar acrosin. Biol Reprod $32,619-63$

Dunbar BS, Maresh GA, Washenik K (1989) Ovarian development and the formation of the mammalian zone pellucida. In: The Mammalian Egg Coat. Structure and Function (J Dietl, ed). Springer-Verlag, Berlin, 38-42

Florman HM, First NL (1988) The regulation of acrosomal exocytosis. Dev Biol 128, 453-463

Gandolfi F, Modina S, Brevini TAL, Galli C, Moor RM, Lauria A (1991) Oviduct ampullary 
epithelium contributes a glycoprotein to the zona pellucida, perivitelline space and blastomeres membrane of sheep embryos. Eur J Bas Appl Histochem 35, 383-392

Greve JM, Salzmann GS (1982) Biosynthesis of the major zona pellucida glycoprotein secreted by oocytes during mammalian oogenesis. Cell 31, 749-759

Greve JM, Salzmann GS, Roller RJ, Wassarman PM (1982) Biosynthesis of the major zona pellucida glycoprotein secreted by oocytes during mammalian oogenesis. Cell 31 , 749-759

Greve LC, Prody GA, Hedrick J (1985) N-acetyl $\beta$-D-glucosaminidase activity in the cortical granules of Xenopus laevis eggs. Gamete Res 12, 305-312

Gwatkin RBL, Williams DT, Carlo DJ (1977) Immunization of mice with heat-solubilized hamster zonae: production of anti-zona antibody and inhibition of fertility. Fertil Steril 28 , 871-877

Gwatkin RBL, Andersen OF, Williams DT (1980) Large scale isolation of bovine and pig zonae pellucidae: chemical, immunological, and receptor properties. Gamete Res 3, 217-231

Hartmann JF (1983) Mammalian fertilization: gamete surface interaction in vitro. In: Mechanism and Control of Animal Fertilization (JF Hartmann, ed). Academic Press, New York, 325-363

Hedrick JL, Wardrip NJ (1986) Isolation of the zona pellucida and purification of its glycoprotein families pig oocytes. Anal Biochem 157, 63-70

Hedrick JL, Wardrip NJ (1987a) On the macromolecular composition of the zona pellucida from porcine oocyte. Dev Biol 121, 478-488

Hedrick JL, Wardrip NJ, Berger T (1987b) Differences in the macromolecular composition of the zona pellucida isolated from pig oocytes, eggs and zygotes. J Exp Zool 241, 257-262

Isojima S, Koyama K, Hasegawa A, Tsunoda $Y$, Hanada A (1984) Monoclonal antibodies to porcine zona pellucida antigen and their inhibitory effects on fertilization. J Reprod I $\mathrm{mmu}$ nol $6,77-87$

Kapur RP, Johnson LV (1988) Ultrastructural evidence that specialized regions of the murine oviduct contribute a glycoprotein to the extracellular matrix of mouse oocyte. Anat Rec 221, 720-729
Katska L, Kauffold P, Smorag Z, Duschinski U, Torner H, Kanitz W (1989) Influence of hardening of the zona pellucida on in vitro fertilization of bovine oocytes. Theriogenology 32 (5), 767-777

Koyama D, Hasegawa $A$, Inoue $M$, Isojima $S$ (1991) Blocking of human sperm-zona interaction by monoclonal antibodies to a glycoprotein family (ZP4) of porcin zona pellucida. Biol Reprod 45, 727-735

Laemmli UK (1970) Cleavage of structural proteins during the assembly of the head of bacteriophage, T4. Nature 227, 680-685

Maresh GA, Dunbar BS (1987) Antigenic comparison of five species of zona pellucida from fertilized and unfertilized eggs. $J$ Reprod Fertil $82,151-161$

Mintz B (1962) Experimental study of the developing mammalian egg: removal of the zona pellucida. Science 138, 594-595

Modlinski JA (1970) The role of the zona pellucida in the development of mouse eggs in vivo. $J$ Embryol Exp Morphol 23, 539-547

Moller CC, Wassarman PM (1989) Characterization of a proteinase that cleaves zona peliucida glycoprotein ZP2 following activation of mouse eggs. Dev Biol 132, 103-112

Mori $\mathrm{T}$, Kamada M, Yamano $\mathrm{S}$, Konishita $\mathrm{T}$, Kano K, Mori T (1985) Production of monoclonal antibodies to porcine zona pellucida and their inhibition of sperm penetration through human zona pellucida in vitro. J $R e$ prod Immunol 8, 1-11

Morrissey $\mathrm{JH}$ (1981) Silver stain for proteins in polyacrylamide gels: a modified procedure with enhanced uniform sensitivity. Anal Biochem 117, 307-310

O'Farrell PH (1975) High resolution twodimensional electrophoresis of proteins. J Biol Chem 250, 4007-4021

Roux E, Kan FWK (1991) Changes of glycoconjugates contents of the zona pellucida during oocyte growth and development in the golden hamster: a quantitative cytochemical study. Anat Rec 230, 347-360

Sacco AG, Yurewicz EC, Subramanian MG, De Mayo JF (1981) Zona pelfucida composition: species cross-reactivity and contraceptive potential of antiserum to a purified pig zona antigen (PPZA). Biol Reprod 25, 997-1108

Shabanowitz RB, O'Rand MG (1988) Characterization of the human zona pellucida from fer- 
tilized and unfertilized eggs. $J$ Reprod Fert 82, 151-161

Shapiro SS, Brown NE, Yard AS (1974) Isolation of an acidic glycoprotein from rabbit oviductal fluid and its association with the egg coating. J Reprod Fertil 40, 281-290

Smith PK, Krohn RI, Hermanson GT, Mallia AK, Gartner FH, Provenzano MD, Fujimoto EF, Goeke NM, Olson BJ, Klenk DC (1985) Measurement of protein using bicinchoninic acid. Anal Biochem 150, 76-85

Smorag Z, Katska $L$ (1988) Reversible changes in dissolution of the zona pellucida of immature bovin oocytes. Theriogenology 30 (1), 13-22

Stewart-Savage J, Bavister BD (1988) A cell surface block to polyspermy occurs in the golden hamster eggs. Dev Biol 128, 150-157
Wassarman PM (1988) Zona pellucida glycoproteins. Ann Rev Biochem 57, 415-442

Wassarman PM, Mortillo $S$ (1991) Structure of the mouse egg extracellular coat, the zona pellucida. Int Rev Cytol 130, 85-110

Wegner CC, Killian GJ (1991) In vitro and in vivo association of an oviductal estrusassociated protein with bovine zona pellucida. Mol Reprod Dev 29, 77-84

Wolgemuth DJ, Celenza J, Bundman DS, Dunbar BS (1984) Formation of the rabbit zona peliucida and its relationship to ovarian follicular development. Dev Biol 106, 1-14

Zaccharius RM, Zell TE, Morrison JH, Woodlock JJ (1969) Glycoprotein staining following electrophoresis on acrylamide gels. Anal Biochem $30,148-152$ 